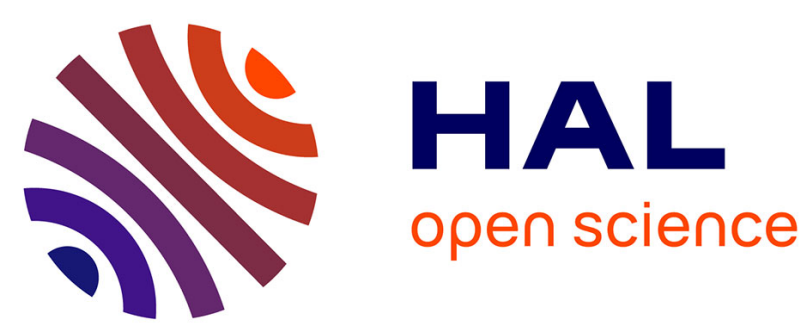

\title{
TROPICAL AND MORPHOLOGICAL OPERATORS FOR SIGNALS ON GRAPHS
}

Samy Blusseau, Santiago Velasco-Forero, Jesus Angulo, Isabelle Bloch

\section{To cite this version:}

Samy Blusseau, Santiago Velasco-Forero, Jesus Angulo, Isabelle Bloch. TROPICAL AND MORPHOLOGICAL OPERATORS FOR SIGNALS ON GRAPHS. 2018 25th IEEE International Conference on Image Processing (ICIP), Oct 2018, Athens, Greece. 10.1109/ICIP.2018.8451395 . hal-01799029v2

\section{HAL Id: hal-01799029 \\ https://hal.science/hal-01799029v2}

Submitted on 1 Jun 2018

HAL is a multi-disciplinary open access archive for the deposit and dissemination of scientific research documents, whether they are published or not. The documents may come from teaching and research institutions in France or abroad, or from public or private research centers.
L'archive ouverte pluridisciplinaire HAL, est destinée au dépôt et à la diffusion de documents scientifiques de niveau recherche, publiés ou non, émanant des établissements d'enseignement et de recherche français ou étrangers, des laboratoires publics ou privés. 


\title{
TROPICAL AND MORPHOLOGICAL OPERATORS FOR SIGNALS ON GRAPHS
}

\author{
Samy Blusseau ${ }^{\star}$, Santiago Velasco-Forero ${ }^{\star}$, Jesús Angulo ${ }^{\star}$, Isabelle Bloch ${ }^{\dagger}$ \\ * CMM, Mines ParisTech, PSL Research University, Paris, France \\ † LTCI, Télécom ParisTech, Université Paris-Saclay, Paris, France
}

\begin{abstract}
We extend recent work on mathematical morphology for signal processing on weighted graphs, based on discrete tropical algebra. The framework is general and can be applied to any scalar function defined on a graph. We show applications in structure tensors analysis and the regularisation of greyscale images.
\end{abstract}

Index Terms - Mathematical morphology, signal processing on graphs, tropical algebra.

\section{INTRODUCTION}

Classical signal and image processing uses the natural geometry of the space supporting the signal (often Euclidean, or a regular discrete set) to define analysis operators such as differentiation, convolution, Fourier transform, interpolation. Processing a function supported by a graph requires to overcome the lack of regular geometrical structure, which leaves us without concepts as simple as translation, for example. Lately, signal processing on graphs has gained interest to address this kind of issues [1, 2, 3]. This effort includes the extension of non-linear operators, and in particular mathematical morphology, to signals on graphs [4].

Mathematical morphology (MM) first arose as a branch of non-linear image processing [5] and since then has been extended to a general mathematical framework based on lattice theory [6]. The formulation of morphological operators on graphs is a classical quest, but the previous formulations were mainly focused on unweighted graphs (use of graph structuring elements) [7] or based on $\mathrm{max} / \mathrm{min}$ operations between vertices and edges weights [8]. The latter is appropriate to formulate watershed segmentation as a graph transform.

In [4] the authors introduce a formalism for MM for signals on graphs that generalises a wide range of mathematical morphology operators usually defined for images, including flat, non-flat and adaptive erosions and dilations. This formalism is clearly inspired by the idea of non-local morphology [9, 10]. Interestingly, although in [4] the authors do not emphasise this aspect, they write dilations and erosions as max-plus and min-plus "linear" functions, in other words as the product of a matrix by a vector in the "max-plus" and "min-plus" algebras, also called idempotent or tropical alge- bras [11]. This is just a hint of how much the field of idempotent mathematics can be suited to the processing of signals on graphs from a mathematical morphology perspective. There are indeed strong links between idempotent algebras and optimal paths computations in graphs [12], as well as between these algebras and mathematical morphology [13, 14, 15, 16].

The goal of this paper is twofold. First, we introduce a general framework for morphological signal processing on graphs, using a tropical algebraic formulation. This opens the very rich field of max-plus algebra [17] to image processing and mathematical morphology. Secondly, we show two examples of applications based on the structure tensors of 2D images. One is the processing of the structure tensors anisotropy for the enhancement of fibre-like structures such as vessels. The second application is the morphological regularisation of greyscale images.

\section{SETTING AND DEFINITIONS}

\subsection{Discrete max-plus algebra}

We consider the semiring $\mathbb{R}_{\max }=\mathbb{R} \cup\{-\infty\}$ equipped with the two binary operations $x \oplus y=\max (x, y)$ and $x \otimes y=$ $x+y$. The neutral element for $\oplus$ is $-\infty(\max (x,-\infty)=x)$, which is also absorbing for $\otimes(x+(-\infty)=-\infty)$, and the neutral element for $\otimes$ is 0 . What is more, both operations are associative and commutative, and $\otimes$ is distributive over $\oplus$. Therefore in this setting the $\oplus$ operation is the counterpart of the addition in the usual algebra, whereas $\otimes$ is analogous to the multiplication. However one specificity of $\left(\mathbb{R}_{\max }, \oplus, \otimes\right)$ is the idempotency of the $\oplus(\max (x, x)=x)$, for which it is called an idempotent semiring.

The set of matrices with elements in $\mathbb{R}_{\max }$ also have their natural "max-plus" counterpart of linear operations, based on $\oplus$ and $\otimes$. Let $A$ and $B$ be two $m \times n$ and $n \times p$ matrices, respectively. Then $A \odot B$ is the $m \times p$ matrix defined by $(A \odot B)_{i j}=\oplus_{k=1}^{n} a_{i k} \otimes b_{k j}=\max _{1 \leq k \leq n}\left(a_{i k}+b_{k j}\right)$. For two matrices of the same size we also have the $\oplus$ operation defined by $(A \oplus B)_{i j}=a_{i j} \oplus b_{i j}=\max \left(a_{i j}, b_{i j}\right)$.

In the remaining of the paper we will note $A^{p}$ the $p$-th power of a square matrix $A$ in the max-plus sense, for any integer $p \geq 0$ (for $p=0, A^{p}$ is the max-plus identity matrix, for which $a_{i i}=0$ and $a_{i j}=-\infty$ for $i \neq j$ ). The coefficients 
of the matrix $A^{p}$ will be noted $a_{i j}^{(p)}$. Finally, we will note $\operatorname{Mat}_{n}\left(\mathbb{R}_{\max }\right)$ the set of $n \times n$ matrices with elements in $\mathbb{R}_{\max }$. In this paper we focus on a particular class of square matrices, defined hereafter.

Definition 1 (Conservative morphological weight matrix [4]) A matrix $W \in$ Mat $_{n}\left(\mathbb{R}_{\max }\right)$ is a conservative morphological weight matrix if $-\infty \leq w_{i j} \leq 0$ and $w_{i i}=0$ for any $(i, j), 1 \leq i, j \leq n$.

\subsection{Graphs with conservative morphological weights}

Let $G=(V, E)$ be a weighted and directed graph containing $n$ vertices, whose $n \times n$ adjacency matrix, noted $W$, is a conservative morphological weight matrix (see Def. 1). We now recall and adapt a few definitions from graph theory.

A path from vertex $i$ to vertex $j$ in $G$ is a tuple of vertices $\left(k_{1}, \ldots, k_{l}\right)$ such that $k_{1}=i, k_{l}=j$, and $\left(k_{p}, k_{p+1}\right) \in E$ for $1 \leq p \leq l-1$. We note $\Gamma_{i j}$ the set of paths from $i$ to $j$ in $G$, and $\Gamma_{i j}^{(p)}$ the set of paths from $i$ to $j$ in $G$ containing at most $p$ vertices, $p \geq 1$. Given the weight matrix $W$, the weight of a path $\gamma=\left(k_{1}, \ldots, k_{l}\right)$, noted $\omega(\gamma)$, is the sum $\omega(\gamma)=\sum_{p=1}^{l-1} w_{k_{p} k_{p+1}}$. One can easily show that the set $\left\{\omega(\gamma), \gamma \in \Gamma_{i j}\right\}$ has a (finite) maximum value whenever $\Gamma_{i j}$ is non empty. We call maximal weight and note $\omega^{*}(\Gamma)$ this value and a maximal path is a path achieving it. For completeness, we set $\omega^{*}(\emptyset)=-\infty$.

The following results show some strong links between tropical algebra and graphs. They can be found in various forms in the literature (e.g. $[12,18]$ ) hence the proof is omitted here. We recall that the powers $W^{p}$ are intended in the max-plus sense (Section 2.1).

Proposition 1 Let $p \in \mathbb{N}$. Then for any $1 \leq i, j \leq n$, 1. $w_{i j}^{(p)}>-\infty$ if and only if there is at least a path from vertex $i$ to vertex $j$ in $G$ containing at most $p+1$ vertices; 2. $w_{i j}^{(p)}$ is the maximal weight of the set of paths from vertex $i$ to vertex $j$ containing at most $p+1$ vertices.

Interestingly, $W^{p}$ is also a conservative morphological weight matrix. Its corresponding graph, that we note $G^{p}$, can be seen as the original graph $G$ to which an edge is added between vertices $i$ and $j$ whenever there is a path containing at most $p+1$ vertices from $i$ to $j$. The weight associated with this new edge is $w_{i j}^{(p)}=\omega^{*}\left(\Gamma_{i j}^{(p+1)}\right)$, the maximal weight for the paths from $i$ to $j$ containing at most $p+1$ vertices. The following corollary is straightforward from the graph interpretation of the max-plus powers of $W$, given by Proposition 1 .

Corollary $1 W^{n}=W^{n-1}$ and therefore there exists an integer $p_{\max }=\min \left\{p \in \mathbb{N}, W^{p}=W^{p+1}\right\}$.

Recall that $n$ is the number of columns (or rows) of $W$. We will note $W^{\infty} \doteq W^{p_{\max }}=W^{n-1}$. It is clear from Proposition 1 that $w_{i j}^{(\infty)}>-\infty$ if and only if there is a path from $i$ to $j$, and $w_{i j}^{(\infty)}$ is the maximal weight over the paths from $i$ to $j: w_{i j}^{(\infty)}=\omega^{*}\left(\Gamma_{i j}\right)$. What is more, one can easily check that $W^{p+1} \geq W^{p}$ and therefore $W^{\infty}=\oplus_{p \geq 0} W^{p}$ is the closure (or Kleene star [19]) of $W$, also noted $W^{*}$.

\subsection{Morphological operators}

In this section we link the max-plus product $W \odot \mathbf{x}$ (Section 2.1) to morphological operators on a vector $\mathrm{x}$ supported by a graph $G$ with conservative morphological weights (Section 2.2). This formulation, that will be summarised by Equation 3 (see infra) generalises many of the dilations and erosions defined on graphs and in image processing applications.

Mathematical morphology is usually defined on complete lattices [20]. A complete lattice $(L, \leq)$ is a partially ordered set for which every subset $A \subseteq L$ has a supremum noted $\bigvee A$ and an infimum noted $\bigwedge A$. A mapping $\delta: L \rightarrow L$ is a dilation if it commutes with the supremum, and an erosion $\varepsilon$ is an operator that commutes with the infimum: for a family $\left(\mathbf{x}_{k}\right)_{k \in K}$ of $L, \delta\left(\bigvee_{k \in K} \mathbf{x}_{k}\right)=\bigvee_{k \in K} \delta\left(\mathbf{x}_{k}\right)$ and $\varepsilon\left(\bigwedge_{k \in K} \mathbf{x}_{k}\right)=\bigwedge_{k \in K} \varepsilon\left(\mathbf{x}_{k}\right)$, where $K$ is any index set.

We recall that $W$ denotes a $n \times n$ conservative morphological weight matrix. We note $L=\left([0,1]^{n}, \leq\right)$ the complete lattice equipped with the usual product partial ordering (Pareto ordering): $\mathbf{x} \leq \mathbf{y} \Longleftrightarrow x_{i} \leq y_{i} \quad \forall i \in\{1, \ldots, n\}$. The supremum and infimum on $L$ are induced by the Pareto ordering: for a family $\left(\mathbf{x}^{(k)}\right)_{k \in K}$ of $L, \bigvee_{k \in K} \mathbf{x}^{(k)}$ is the vector $\mathbf{y}$ defined by $y_{i}=\bigvee_{k \in K} x_{i}^{(k)}$, which may not be any of the $\mathbf{x}^{(k)}$ (and the same holds for $\Lambda$ ). Then the "max-pluslinear" function

$$
\delta_{W}:\left\{\begin{array}{llc}
L & \rightarrow & L \\
\mathbf{x} & \mapsto & W \odot \mathbf{x}
\end{array}\right.
$$

is a dilation on $L$. Similarly, noting $\mathbf{x}^{c}=1-\mathbf{x}$,

$$
\varepsilon_{W}(\mathbf{x})=\left(W^{T} \odot \mathbf{x}^{c}\right)^{c}
$$

is an erosion on $L$ and the pair $\left(\varepsilon_{W}, \delta_{W}\right)$ forms an adjunction: $\forall \mathbf{x}, \mathbf{y} \in L, \quad \delta_{W}(\mathbf{x}) \leq \mathbf{y} \Longleftrightarrow \mathbf{x} \leq \varepsilon_{W}(\mathbf{y})$.

Let $\mathbf{x}=\left(x_{1}, \ldots, x_{n}\right) \in L$ be a signal on $G$, meaning that each value $x_{i}$ is supported by vertex $i$. Then the dilation and erosion of Equations 1 and 2 can be written for $1 \leq i \leq n$

$\delta_{W}(x)_{i}=\max _{j \in \mathcal{N}_{i}}\left(x_{j}+w_{i j}\right), \quad \varepsilon_{W}(x)_{i}=\min _{j, i \in \mathcal{N}_{j}}\left(x_{j}-w_{j i}\right)$

where $\mathcal{N}_{i} \doteq\{j \in\{1, \ldots, n\},(i, j) \in E\}$ is the set of neighbours of vertex $i$ in $G$. At this point one should remember that the $w_{i j}$ are non positive. The closer $w_{i j}$ is to 0 , the more likely vertex $j$ is to contribute to the $\max$ (resp. the min) in the dilation (resp. erosion) of vertex $i$. Conversely, the closer $w_{i j}$ is to $-\infty$, the more likely vertex $j$ is to be irrelevant in these computations. In fact, the max can be taken over $\mathcal{N}_{i}$ in the dilation because $w_{i j}=-\infty$ if $j \notin \mathcal{N}_{i}$, and the same holds 


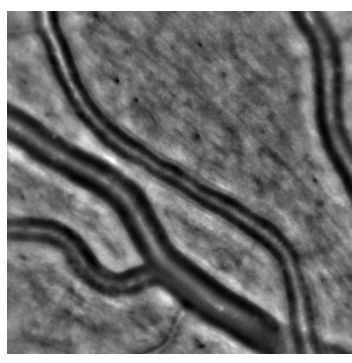

(a)

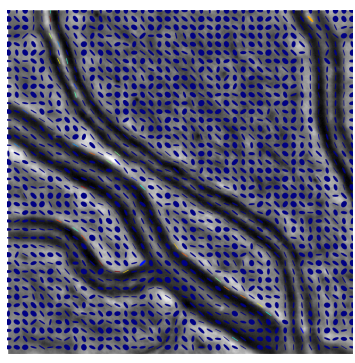

(b)
Fig. 1. (a) A greyscale image and (b) its structure tensors $T_{\sigma}$ for $\sigma=3$ pixels, obtained with [21]. The corresponding anisotropy image is shown in Figure 3 top left.

for the erosion. The dilation thus appears as a max-plus "nonlocal" convolution where coefficients $w_{i j}$ model the weight of the neighbouring vertices in the graph. Note that Equation 3 generalises many adjunctions in image processing. For example, setting $w_{i j}=\log \left(\mathbb{1}_{\mathcal{B}_{i}}(j)\right)$ with $\mathcal{B}_{i}$ a structuring element for pixel $i$, we get the usual flat dilation and erosion. If the family $\left(\mathcal{B}_{i}\right)_{i \in I}$ is not translation invariant, we get the so called adaptive morphology framework; if $\mathcal{B}_{i}$ is not a spatial neighbourhood of $i$, we get the typical non-local operators.

As it is well known in mathematical morphology, if a dilation $\delta$ and an erosion $\varepsilon$ form an adjunction, then the dilation $\delta^{p}=\delta \circ \cdots \circ \delta$ and the erosion $\varepsilon^{p}=\varepsilon \circ \cdots \circ \varepsilon$ as well. The associativity of the max-plus product $\odot$ yields the following result for these iterated dilation and erosion.

Proposition 2 Let $W$ a conservative morphological weight matrix, and $p \in \mathbb{N}$. Then $\delta_{W}^{p}=\delta_{\left(W^{p}\right)}$ and $\varepsilon_{W}^{p}=\varepsilon_{\left(W^{p}\right)}$.

This also implies the semi-group property $\delta_{\left(W^{p}\right)} \delta_{\left(W^{q}\right)}=$ $\delta_{\left(W^{p+q}\right)}$, and an easy writing for $\delta_{W}^{p}$ and $\varepsilon_{W}^{p}$ :

$\delta_{W}^{p}(x)_{i}=\max _{j \in \mathcal{N}_{i}^{p}}\left(x_{j}+w_{i j}^{(p)}\right), \quad \varepsilon_{W}^{p}(x)_{i}=\min _{j, i \in \mathcal{N}_{j}^{p}}\left(x_{j}-w_{j i}^{(p)}\right)$

where $\mathcal{N}_{i}^{p}$ is the set of neighbours of vertex $i$ in $G^{p}$ or, equivalently, the set of vertices in $G$ that can be reached from $i$ through a path containing at most $p+1$ vertices.

Since $W^{p}$ is a conservative morphological weight matrix for any $p$, it is clear from Equation 4 that $\delta_{W}^{p}$ is extensive and $\varepsilon_{W}^{p}$ is anti-extensive. From Corollary 1 and Proposition 2 we get the idempotence of $\delta_{W}^{\infty} \doteq \delta_{\left(W^{\infty}\right)}$ and $\varepsilon_{W}^{\infty} \doteq \varepsilon_{\left(W^{\infty}\right)}$, which are therefore a closing and an opening respectively, that verify $\delta_{W}^{\infty} \varepsilon_{W}^{\infty}=\varepsilon_{W}^{\infty}$ and $\varepsilon_{W}^{\infty} \delta_{W}^{\infty}=\delta_{W}^{\infty}$.

\section{APPLICATION TO STRUCTURE TENSORS}

In this section we introduce a class of graphs based on the co-circularity of the structure tensors principal directions. Structure tensors and anisotropy image [21]. Given an image $f$, the scale zero tensor $T_{0}$ maps each pixel $(x, y)$ to the symmetric positive semi-definite matrix with rank $\leq 1$,

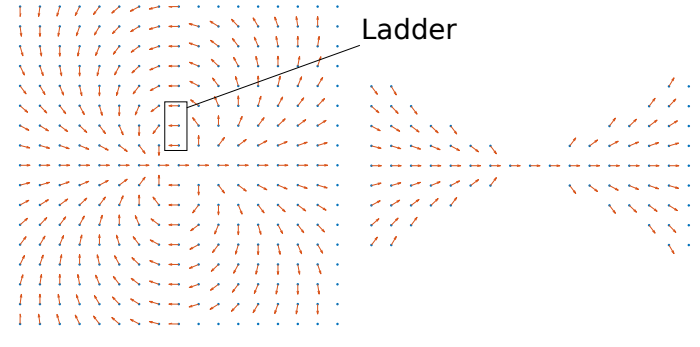

Fig. 2. Left: a set of elements $(u, \theta)$ co-circular to the central element $\left(u_{0}, \theta_{0}\right)$ where $u_{0}$ is the origin and $\theta_{0}=0$ rad; Right: the elements from the left hand image that comply with the additional constraint to avoid ladder configurations.

$T_{0}(x, y)=\nabla f(x, y) \cdot \nabla f(x, y)^{T}$. The gradient $\nabla f(x, y)$ is eigenvector of $T_{0}(x, y)$ with $\|\nabla f(x, y)\|^{2}$ as corresponding eigenvalue, and 0 is the other eigenvalue. Then for $\sigma>0$ the tensor $T_{\sigma}$ is the smoothed version $T_{\sigma}=G_{\sigma} * T_{0}$ where $G_{\sigma}$ is the $\sigma$-scale Gaussian kernel. Note that, for any $u=(x, y), T_{\sigma}(u)$ is a weighted sum of tensors $T_{0}(v)$ with positive weights, and is therefore a positive semi-definite matrix that can be represented by an ellipse (Fig. 1(b)). We note $0 \leq \lambda_{2}(u) \leq \lambda_{1}(u)$ the eigenvalues of $T_{\sigma}(u)$ and $\left(e_{1}(u), e_{2}(u)\right)$ its basis of orthogonal eigenvectors. If we represent $T_{\sigma}(u)$ by its corresponding ellipse then its main direction is given by the second eigenvector $e_{2}(u)$. We note $\theta_{2}(u)=\arg \left(e_{2}(u)\right)$. From the tensor field $T_{\sigma}$ we can build the anisotropy image $I_{a}=\frac{\lambda_{1}-\lambda_{2}}{\lambda_{1}+\lambda_{2}}$. Then $0 \leq I_{a} \leq 1$, and the closer $I_{a}(u)$ to 1 , the more $T_{\sigma}(u)$ is anisotropic (Fig. 3).

Co-circularity (Fig. 2). Given two points $u_{1}, u_{2} \in \mathbb{R}^{2}$ and two angles $\theta_{1}, \theta_{2}$ we say that $\left(u_{1}, \theta_{1}\right)$ and $\left(u_{2}, \theta_{2}\right)$ are co-circular if there is a circle (with possibly infinite radius) tangent in $u_{1}$ and $u_{2}$ to the lines directed by the vectors $\left[\cos \left(\theta_{1}\right), \sin \left(\theta_{1}\right)\right]^{T}$ and $\left[\cos \left(\theta_{2}\right), \sin \left(\theta_{2}\right)\right]^{T}$, respectively.

An undirected graph with binary weights. The graph $G$ we consider is built for an image containing $n$ pixels. It is undirected and contains $n$ vertices, one per pixel. Noting $W$ its adjacency matrix, we set $w_{i j}=0$ iff the point $u_{j}$ is in a square window of fixed size $2 k+1$, centered on $u_{i}$, and $\left(u_{i}, \theta_{2}(i)\right)$ and $\left(u_{j}, \theta_{2}(j)\right)$ are co-circular up to a certain angular tolerance $\alpha$, with an additional constraint to avoid ladder configurations (Figure 2). Otherwise, $w_{i j}=-\infty$.

\subsection{Processing anisotropy}

Processing an array of non-scalar data containing directional information is a complex task which can be of crucial interest. An example is tractography, which consists in tracking white matter fibres in 3D diffusion MRI of the brain [22]. Another possible application is the analysis of thin structures such as vessels in $2 \mathrm{D}$ images, through their sets of structure tensors (Figure 1 (b)). We present a method for the latter application, based on the operators defined in the previous sections.

As there is no meaningful ordering defining a complete 
lattice on positive semi-definite matrices, we choose to perform a morphological analysis on scalar data inferred from the matrices. Thus we define the co-circularity graph as described above and the processed signal $x_{i}$ on vertex $i$ is the anisotropy of the structure tensor at pixel $i$. As Figure 3 shows (top row), anisotropy can be high even outside the relevant structures, and thresholding the image would not help identify the vessel. Our aim is to keep the anisotropy as high as possible in the vessels, while reducing it in the background. Figure 3 shows the resulting anisotropy after application of
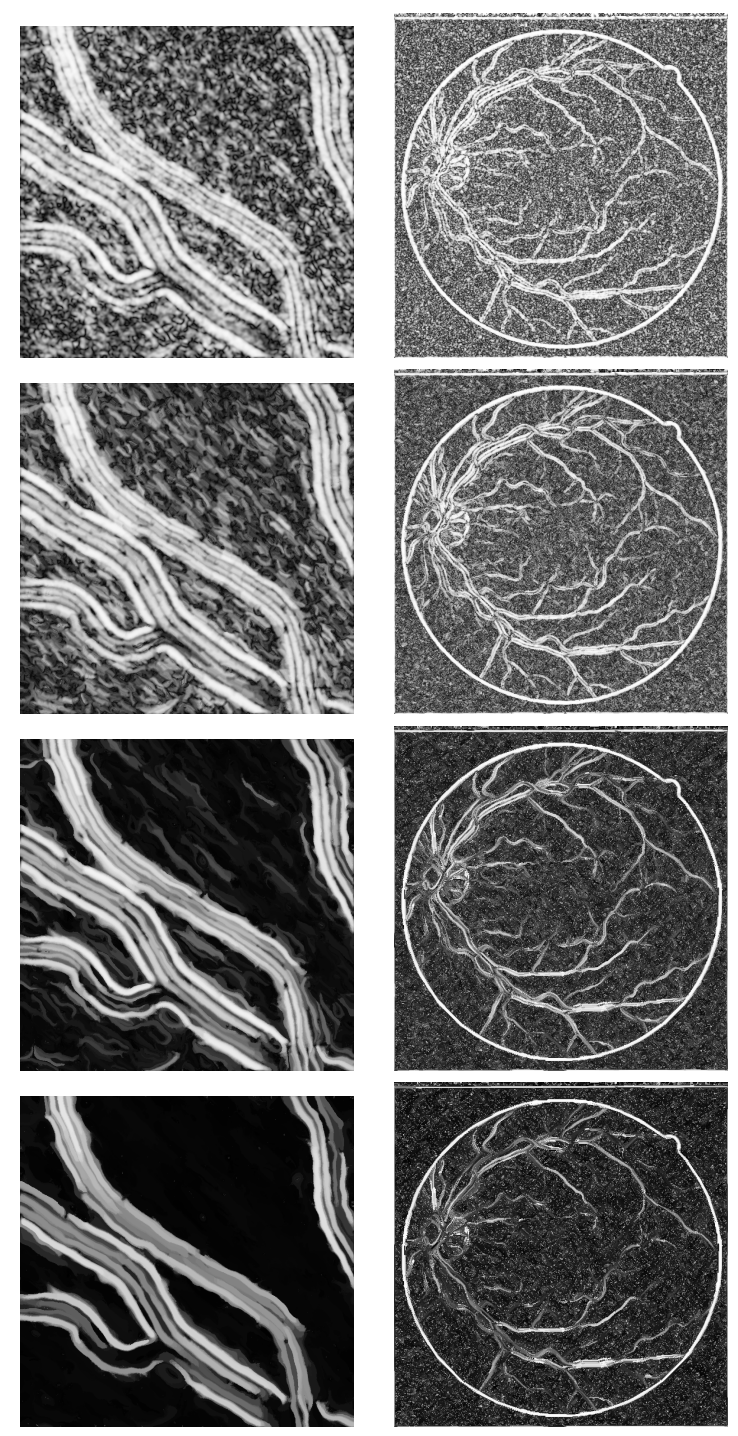

Fig. 3. Examples of openings $\gamma_{W}^{(p)}$ on anisotropy images, in the case of a binary and symmetric matrix $W$, computed as explained in Section 3. For each column, from top to bottom: $p=0$ (original anisotropy image), $p=1,5,10$.

openings $\gamma_{W}^{(p)} \doteq \delta_{W}^{p} \varepsilon_{W}^{p}$ for different values of $p$. As expected, for an appropriate $p$, the opening keeps brighter those vertices that are part of large and bright connected components in the
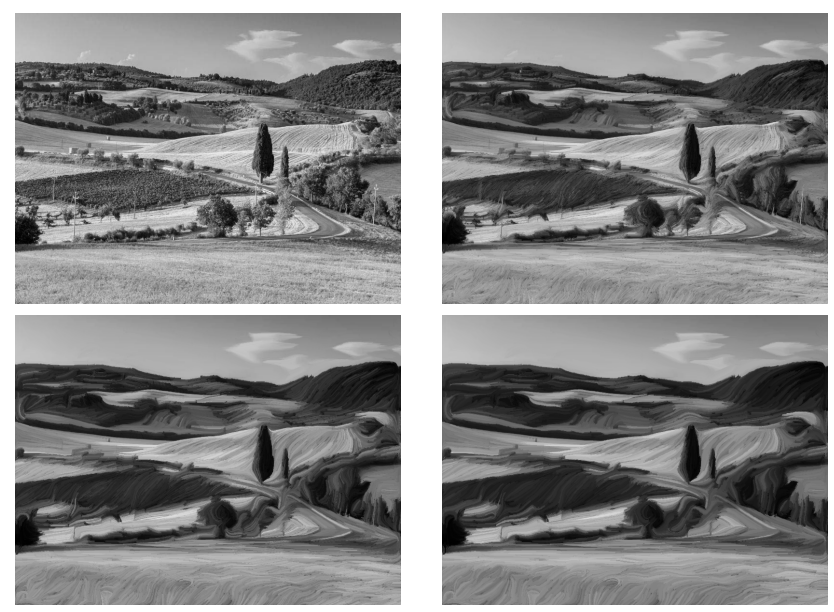

Fig. 4. Examples of openings $\gamma_{W}^{(p)}$ on a natural image, with $W$ a binary and symmetric matrix, computed from the structure tensors as explained in Section 3. From top to bottom and left to right: $p=0$ (original image), $p=1,2,3$.

sense of the co-circularity graph. What is more, it produces homogeneous regions that match the structure of the vessels. This could be a useful pre-processing that could improve both segmentation and quantification.

\subsection{Morphological anisotropic diffusion}

Once the co-circularity graph is computed from the structure tensors, the operators defined earlier can also be applied to the original greyscale image, instead of doing so on the anisotropy image. This produces a regularising effect preserving the most salient edges. Figure 4 is an example of openings $\gamma_{W}^{(p)}$ applied to a landscape picture. Note that $\gamma_{W}^{(p)}$ is an idempotent operator, meaning that once $p$ is chosen, the regularisation effect is obtained once and for all after one opening.

\section{CONCLUSION}

We introduced a framework that generalises mathematical morphology for signals on graphs, and that relies on a maxplus algebra formulation. This opens several questions such as the links between the properties of the weight matrix and those of the morphological operators. These questions shall be investigated with the support of the rich existing literature on idempotent mathematics and graphs, and also from the perspective of applications. The operators we defined based on the co-circularity of structure tensors show interesting behaviours for the processing of anisotropic structures and the morphological regularisation of natural images. However, further work is needed, as for example the development of a multi-scale approach or an automatic scale determination, as well as comparison with other methods. 


\section{REFERENCES}

[1] D. I. Shuman, S. K. Narang, P. Frossard, A. Ortega, and P. Vandergheynst, "The emerging field of signal processing on graphs: Extending high-dimensional data analysis to networks and other irregular domains," IEEE Signal Processing Magazine, vol. 30, no. 3, pp. 83-98, May 2013.

[2] F. Lozes and A. Elmoataz, "Nonlocal difference operators on graphs for interpolation on point clouds," in International Symposium on Mathematical Morphology and Its Applications to Signal and Image Processing. Springer, 2017, pp. 309-316.

[3] G. Puy, S. Kitic, and P. Pérez, "Unifying local and nonlocal signal processing with graph CNNs," CoRR, vol. abs/1702.07759, 2017.

[4] S. Velasco-Forero and J. Angulo, "Nonlinear operators on graphs via stacks," in Geometric Science of Information, Proceedings, F. Nielsen and F. Barbaresco, Eds. 2015, pp. 654-663, Springer International Publishing.

[5] J. Serra, Image analysis and mathematical morphology, Academic Press, Inc., 1982.

[6] I. Bloch, H.J.A.M. Heijmans, and C. Ronse, "Mathematical morphology," in Handbook of Spatial Logics, M. Aiello, I. Pratt-Hartmann, and J. Van Benthem, Eds., pp. 857-944. Springer Netherlands, Dordrecht, 2007.

[7] H.J.A.M. Hejmans, P. Nacken, A. Toet, and L. Vincent, "Graph morphology," Journal of Visual Communication and Image Representation, vol. 3, no. 1, pp. 24 - 38, 1992.

[8] L. Najman and J. Cousty, "A graph-based mathematical morphology reader," Pattern Recognition Letters, vol. 47, pp. 3 - 17, 2014, Advances in Mathematical Morphology.

[9] P. Salembier, "Study on nonlocal morphological operators," in 16th IEEE International Conference on Image Processing (ICIP), 2009, pp. 2269-2272.

[10] S. Velasco-Forero and J. Angulo, "On nonlocal mathematical morphology," in International Symposium on Mathematical Morphology and Its Applications to Signal and Image Processing. Springer, 2013, pp. 219-230.

[11] G. L. Litvinov, "Maslov dequantization, idempotent and tropical mathematics: A brief introduction," Journal of Mathematical Sciences, vol. 140, no. 3, pp. 426-444, Jan 2007.

[12] B. A. Carré, "An algebra for network routing problems," IMA Journal of Applied Mathematics, vol. 7, no. 3, pp. 273-294, 1971.
[13] P. Del Moral and M. Doisy, "Maslov idempotent probability calculus. II," Theory of Probability \& Its Applications, vol. 44, no. 2, pp. 319-332, 2000.

[14] B. Burgeth and J. Weickert, "An explanation for the logarithmic connection between linear and morphological system theory," International Journal of Computer Vision, vol. 64, no. 2, pp. 157-169, Sep 2005.

[15] P. Maragos, "Chapter Two - Representations for morphological image operators and analogies with linear operators," vol. 177 of Advances in Imaging and Electron Physics, pp. 45 - 187. Elsevier, 2013.

[16] J. Angulo, "Chapter One - Convolution in (max,min)algebra and its role in mathematical morphology," vol. 203 of Advances in Imaging and Electron Physics, pp. 1 - 66. Elsevier, 2017.

[17] S. Gaubert, "Methods and applications of $(\max ,+)$ linear algebra," in 14th Annual Symposium on Theoretical Aspects of Computer Science (STACS), 1997.

[18] M. Akian, R. Bapat, and S. Gaubert, "Max-plus algebra," Handbook of linear algebra (Discrete Mathematics and its Applications), vol. 39, pp. 10-14, 2006.

[19] S. C. Kleene, "Representation of events in nerve nets and finite automata," Tech. Rep., Rand Project Air Force Santa Monica CA, 1951.

[20] C. Ronse, "Why mathematical morphology needs complete lattices," Signal Processing, vol. 21, no. 2, pp. 129 - 154, 1990.

[21] G. Peyré, "The numerical tours of signal processing," Computing in Science \& Engineering, vol. 13, no. 4, pp. 94-97, 2011.

[22] J.-D. Tournier, F. Calamante, D. G. Gadian, and A. Connelly, "Direct estimation of the fiber orientation density function from diffusion-weighted mri data using spherical deconvolution," NeuroImage, vol. 23, no. 3, pp. 1176 -1185, 2004. 